\title{
ON AN ELLIPTIC EQUATION WITH CONCAVE AND CONVEX NONLINEARITIES
}

\author{
THOMAS BARTSCH AND MICHEL WILLEM
}

(Communicated by Jeffrey B. Rauch)

\begin{abstract}
We study the semilinear elliptic equation $-\Delta u=\lambda|u|^{q-2} u+\mu|u|^{p-2} u$ in an open bounded domain $\Omega \subset \mathbb{R}^{N}$ with Dirichlet boundary conditions; here $1<q<2<p<2^{*}$. Using variational methods we show that for $\lambda>0$ and $\mu \in \mathbb{R}$ arbitrary there exists a sequence $\left(v_{k}\right)$ of solutions with negative energy converging to 0 as $k \rightarrow \infty$. Moreover, for $\mu>0$ and $\lambda$ arbitrary there exists a sequence of solutions with unbounded energy. This answers a question of Ambrosetti, Brézis and Cerami. The main ingredient is a new critical point theorem, which guarantees the existence of infinitely many critical values of an even functional in a bounded range. We can also treat strongly indefinite functionals and obtain similar results for first-order Hamiltonian systems.
\end{abstract}

\section{INTRODUCTION}

We consider the semilinear elliptic problem

$$
\begin{aligned}
-\Delta u & =\lambda|u|^{q-2} u+\mu|u|^{p-2} u & & \text { in } \Omega, \\
u & =0 & & \text { on } \partial \Omega,
\end{aligned}
$$

where $\Omega \subset \mathbb{R}^{N}$ is an open bounded domain with smooth boundary and $1<q<$ $2<p<2^{*}:=2 N /(N-2)$; we set $2^{*}=\infty$ if $N=1,2$. In [1] Ambrosetti et al. showed that for $\lambda>0$ small and $\mu>0$ there exist infinitely many solutions $u \in H_{0}^{1}(\Omega)$ of (1) with negative energy

$$
\phi_{\lambda, \mu}(u):=\frac{1}{2} \int_{\Omega}|\nabla u|^{2} d x-\frac{\lambda}{q} \int_{\Omega}|u|^{q} d x-\frac{\mu}{p} \int_{\Omega}|u|^{p} d x
$$

and infinitely many solutions with positive energy. The goal of this note is to show that the restriction on $\lambda$ is not needed. In fact, we shall prove the following theorem which gives a positive answer to problem (c) of [1].

Theorem 1. Assume that $1<q<2<p<2^{*}$.

(a) For every $\mu>0, \lambda \in \mathbb{R}$, problem (1) has a sequence of solutions $\left(u_{k}\right)$ such that $\phi_{\lambda, \mu}\left(u_{k}\right) \rightarrow \infty$ as $k \rightarrow \infty$.

Received by the editors April 21, 1994.

1991 Mathematics Subject Classification. Primary 58E05; Secondary 34C25, 35J25.

The first author thanks the members of the Départment de Mathématique of the Université Catholique de Louvain for their invitation and hospitality. 
(b) For every $\lambda>0, \mu \in \mathbb{R}$, problem (1) has a sequence of solutions $\left(v_{k}\right)$ such that $\phi_{\lambda, \mu}\left(v_{k}\right)<0$ and $\phi_{\lambda, \mu}\left(v_{k}\right) \rightarrow 0$ as $k \rightarrow \infty$.

We do not know whether $v_{k} \rightarrow 0$ as $k \rightarrow \infty$. This is the case if 0 is the only solution of (1) with energy 0 . However, the following holds.

Proposition 1. (a) For $\lambda \in \mathbb{R}$ and $\mu \leq 0$ there are no solutions with positive energy. Moreover

$$
\inf \left\{\|u\|: u \text { solves }(1), \phi_{\lambda, \mu}(u)>0\right\} \rightarrow \infty \quad \text { as } \mu \rightarrow 0^{+} \text {. }
$$

(b) For $\mu \in \mathbb{R}$ and $\lambda \leq 0$ there are no solutions with negative energy. Moreover

$$
\sup \left\{\|u\|: u \text { solves }(1), \phi_{\lambda, \mu}(u) \leq 0\right\} \rightarrow 0 \text { as } \lambda \rightarrow 0^{+} \text {. }
$$

Theorem 1 remains true if the special nonlinearity in (1) is replaced by an odd function $f(x, u)$ which behaves near 0 asymptotically (and uniformly in $x)$ like $\lambda|u|^{q-2} u, \lambda \neq 0$, and which is superquadratic and subcritical near infinity. In [1] and [7] the existence of solutions with negative energy has also been proved in the critical case $p=2^{*}$ provided $\lambda>0$ is small enough. It is not clear whether this remains true for large $\lambda$ because the local Palais-Smale condition $(\mathrm{PS})_{c}$ fails for large $\lambda$ even if $c<0$; see [7] for a discussion of this.

Whereas the first statement of Theorem 1 can be proved using well-known critical point theorems for even functionals (cf. [2, 3, 8]), we shall need a new critical point theorem in order to obtain the sequence $\left(v_{k}\right)$. Since it does not require more effort, we shall treat more general symmetries than the $\mathbb{Z} / 2$ symmetry in our abstract result and we allow the functional to be strongly indefinite. This allows us to apply our critical point theorem also to first-order Hamiltonian systems having a similar type of nonlinearity.

\section{THE ABSTRACT CRITICAL POINT THEOREM}

Let $X$ be a Banach space and $\varphi \in C^{1}(X, \mathbb{R})$. We are interested in multiple critical points of $\varphi$. For this we need a symmetry condition on $\varphi$ which contains even functionals as a special case. In order to formulate this we have to recall a certain class of admissible representations. Let $G$ be a compact Lie group and $V$ a finite-dimensional orthogonal representation of $G$. Then $V$ is said to be admissible if the following Borsuk-Ulam type condition holds.

Every continuous equivariant map $h: \overline{\mathscr{O}} \rightarrow V^{k}$ where $\mathscr{O}$ is an open, bounded and invariant neighbourhood of 0 in $V^{k+1}, k \geq$ 1 , has a zero in $\partial \mathscr{O}$.

Here $\mathscr{O}$ is invariant if $g v:=\left(g v_{1}, \ldots, g v_{k+1}\right) \in \mathscr{O}$ for every $g \in G$ and $v=\left(v_{1}, \ldots, v_{k+1}\right) \in \mathscr{O}$. The map $h$ is equivariant if $h(g v)=g h(v)$. The classical theorem of Borsuk says that $V=\mathbb{R}$ with the antipodal representation of $G=\mathbb{Z} / 2$ is admissible. Admissible representations can be classified completely using an algebraic criterion (see [4, Theorem 3.7]). From the results of [4] it also follows that admissible representations are precisely those which have the "dimension property" used by Benci [6].

It is clear that an admissible representation $V$ cannot have nontrivial fixed points, that is,

$$
V^{G}:=\{v \in V: g v=v \text { for all } g \in G\}=\{0\} .
$$


We can now state our conditions on $\phi$.

$\left(\mathrm{A}_{1}\right)$ There exists an admissible representation $V$ of $G$ such that $X=$ $\bigoplus_{j \in I} X(j)$ with $I=\mathbb{N}$ or $I=\mathbb{Z}$ and $X(j) \cong V$ for every $j \in I$. The space $X$ is then a Banach space with isometric linear $G$-action. The functional $\phi: X \rightarrow \mathbb{R}$ is invariant under this action: $\phi(g u)=\phi(u)$ for $g \in G$ and $u \in X$.

$\left(\mathrm{A}_{2}\right)$ For every $k \geq k_{0}$ there exists $R_{k}>0$ such that $\phi(u) \geq 0$ for every $u \in X_{k}:=\bigoplus_{j \geq k} X(j)$ with $\|u\|=R_{k}$.

$\left(\mathrm{A}_{3}\right) \quad b_{k}:=\inf _{u \in B_{k}} \varphi(u) \rightarrow 0$ as $k \rightarrow \infty$. Here $B_{k}:=\left\{u \in X_{k}:\|u\| \leq R_{k}\right\}$.

$\left(\mathrm{A}_{4}\right)$ For every $k \geq 1$ there exists $r_{k} \in\left(0, R_{k}\right)$ and $d_{k}<0$ such that $\phi(u) \leq d_{k}$ for every $u \in X^{k}:=\bigoplus_{j \leq k} X(j)$ with $\|u\|=r_{k}$.

$\left(\mathrm{A}_{5}\right)$ Every sequence $u_{n} \in X_{-n}^{n}:=\bigoplus_{j=-n}^{n} X(j)$ with $\phi\left(u_{n}\right)<0$ bounded and $\left(\phi \mid X_{-n}^{n}\right)^{\prime}\left(u_{n}\right) \rightarrow 0$ as $n \rightarrow \infty$ has a subsequence which converges to a critical point of $\phi$.

Observe that $\left(\mathrm{A}_{3}\right)$ and $\left(\mathrm{A}_{4}\right)$ imply $b_{k} \leq d_{k}<0$.

Theorem 2. If $\phi \in C^{1}(X, \mathbb{R})$ satisfies $\left(\mathrm{A}_{1}\right)-\left(\mathrm{A}_{5}\right)$, then for each $k \geq k_{0}, \varphi$ has a critical value $c_{k} \in\left[b_{k}, d_{k}\right]$, hence $c_{k} \rightarrow 0$ as $k \rightarrow \infty$.

Proof. We fix $n \geq k \geq k_{0}$ and define an almost critical value $c_{k}^{n}$ as follows. Setting

$$
X_{k}^{n}:=\bigoplus_{j=k}^{n} X(j)
$$

and

$$
B_{k}^{n}:=\left\{u \in X_{k}^{n}:\|u\| \leq R_{k}\right\}
$$

we define

$$
\Gamma_{k}^{n}:=\left\{\gamma \in \mathscr{C}\left(B_{k}^{n}, X_{-n}^{n}\right): \gamma \text { is equivariant, } \gamma(u)=u \text { if }\|u\|=R_{k}\right\}
$$

and

$$
c_{k}^{n}:=\sup _{\gamma \in \Gamma_{k}^{n}} \min _{u \in B_{k}^{n}} \phi(\gamma(u)) .
$$

We shall show that $b_{k} \leq c_{k}^{n} \leq d_{k}<0$ for every $n \geq k$. By the quantitative deformation lemma (see [10]) $c_{k}^{n}$ is an almost critical value of $\phi \mid X_{-n}^{n}$, that is, there exists a sequence $u_{i} \in X_{-n}^{n}$ with $\phi\left(u_{i}\right) \rightarrow c_{k}^{n}$ and $\left(\phi \mid X_{-n}^{n}\right)^{\prime}\left(u_{i}\right) \rightarrow 0$ as $i \rightarrow \infty$. Using $\left(\mathrm{A}_{5}\right)$ we see that $c_{k}^{n}$ converges along a subsequence to a critical value $c_{k} \in\left[b_{k}, d_{k}\right]$ as $n \rightarrow \infty$.

It remains to prove $c_{k}^{n} \in\left[b_{k}, d_{k}\right]$ for every $n \geq k$. The inequality $c_{k}^{n} \geq b_{k}$ is obvious from the definitions. In order to see $c_{k}^{n} \leq d_{k}$ it suffices to show that for every $\gamma \in \Gamma_{k}^{n}$ there exists $u \in B_{k}^{n}$ satisfying $\gamma(u) \in X^{k}$ and $\|\gamma(u)\|=r_{k}$. Given $\gamma \in \Gamma_{k}^{n}$ we set

$$
\mathscr{O}:=\left\{u \in B_{k}^{n}:\|\gamma(u)\|<r_{k}\right\} \text {. }
$$

The equivariance of $\gamma$ implies $\gamma(0)=0$ because 0 is the only fixed point of the action. Therefore $\mathscr{O}$ is an open invariant neighbourhood of 0 and $\mathscr{O} \subset$ int $B_{k}^{n}$. Let $P: X_{-n}^{n} \rightarrow X_{k+1}^{n}$ be the projection along $X_{-n}^{k}$ and set $h:=P \circ \gamma: \overline{\mathscr{O}} \rightarrow X_{k+1}^{n}$. Since $X_{k}^{n} \cong V^{n-k+1}$ and $X_{k+1}^{n} \cong V^{n-k}$, the admissibility of $V$ gives us a point $u \in \partial \mathscr{\odot}$ with $h(u)=0$. Clearly this implies $\|\gamma(u)\|=r_{k}$ and $\gamma(u) \in X_{-n}^{k} \subset X^{k}$ as required. 
Remark. Theorem 2 can be considered as a dual version of Theorem 2.5 of [3] or Theorem 3.1 of [5].

\section{Proofs}

Proof of Theorem 1. We first prove the existence of $\left(v_{k}\right)$ and assume $\lambda>0$. We set $X:=H_{0}^{1}(\Omega)$ with

$$
\|u\|:=\left(\int_{\Omega}|\nabla u|^{2} d x\right)^{1 / 2} .
$$

Let $\left(e_{j}\right)$ be any orthonormal base of $X$ and set $X(j):=\operatorname{span}\left(e_{j}\right)$. The functional

$$
\begin{aligned}
\phi(u) & :=\frac{1}{2}\|u\|^{2}-\frac{\lambda}{q} \int_{\Omega}|u|^{q} d x-\frac{\mu}{p} \int_{\Omega}|u|^{p} d x \\
& =\frac{1}{2}\|u\|^{2}-\frac{\lambda}{q}\|u\|_{q}^{q}-\frac{\mu}{p}\|u\|_{p}^{p}
\end{aligned}
$$

is well defined on $X$ for $1<q<2<p<2^{*}$. Since $\phi$ is even, assumption $\left(\mathrm{A}_{1}\right)$ is obviously satisfied with $G=\mathbb{Z} / 2$ acting on $V=\mathbb{R}$ via the antipodal map.

In order to see $\left(A_{2}\right)$ we set

$$
\mu_{k}:=\sup _{u \in X_{k}-0}\|u\|_{q} /\|u\| .
$$

It follows easily from the Rellich embedding theorem that $\mu_{k} \rightarrow 0$ as $k \rightarrow \infty$. Choose $c_{1}>0$ such that $\|u\|_{p}^{p} \leq c_{1}\|u\|^{p}$. We obtain for $u \in X_{k}$

$$
\phi(u) \geq \frac{1}{2}\|u\|^{2}-\frac{\lambda}{q} \mu_{k}^{q}\|u\|^{q}-|\mu| \frac{c_{1}}{p}\|u\|^{p} .
$$

Since $p>2$, we have

$$
|\mu| \frac{c_{1}}{p}\|u\|^{p} \leq \frac{1}{4}\|u\|^{2}
$$

for $\|u\| \leq R, R>0$ small. Now we set $R_{k}:=\left(4 \lambda \mu_{k}^{q} / q\right)^{1 /(2-q)}$ so that

$$
\frac{1}{4} R_{k}^{2}=\frac{\lambda}{q} \mu_{k}^{q} R_{k}^{q} \text {. }
$$

Clearly $R_{k} \rightarrow 0$, so there exists $k_{0}$ with $R_{k} \leq R$ when $k \geq k_{0}$. Thus if $u \in X_{k}, k \geq k_{0}$, satisfies $\|u\|=R_{k}$ we have

$$
\phi(u) \geq \frac{1}{4}\|u\|^{2}-\frac{\lambda}{q} \mu_{k}^{q}\|u\|^{q}=0 .
$$

This proves $\left(\mathrm{A}_{2}\right)$. Next $\left(\mathrm{A}_{3}\right)$ follows immediately from $R_{k} \rightarrow 0$. Also $\left(\mathrm{A}_{4}\right)$ is evident because $X^{k}$ is finite dimensional, hence all norms on $X^{k}$ are equivalent. Therefore the term $\frac{-\lambda}{q}\|u\|_{q}^{q}$ dominates near 0 . This is precisely the point where $\lambda>0$ enters. Finally, the Palais-Smale condition $\left(A_{5}\right)$ can be shown as in [8] or [10].

The existence of the sequence $\left(u_{k}\right)$ follows from the symmetric mountain pass theorem of $[2,8]$ or the fountain theorem of $[3,10]$. 
Proof of Proposition 1. (a) Fix $\lambda, \mu \in \mathbb{R}$. From $\phi_{\lambda, \mu}^{\prime}(u)=0$ and $\phi_{\lambda, \mu}(u) \geq 0$ one obtains easily

$$
\left(\frac{1}{2}-\frac{1}{q}\right)\|u\|^{2}+\left(\frac{1}{q}-\frac{1}{p}\right) \mu\|u\|_{p}^{p} \geq 0 .
$$

Since $1<q<2<p$, we see immediately that for $\mu \leq 0$ only $u=0$ is a solution with nonnegative energy. If $\mu>0$, then there are positive constants $c_{1}, c_{2}$ with

$$
-c_{1}\|u\|^{2}+\mu c_{2}\|u\|^{p} \geq 0,
$$

hence

$$
\|u\|^{p-2} \geq \mu^{-1} c_{1} / c_{2} \rightarrow \infty \quad \text { as } \mu \rightarrow 0^{+} .
$$

(b) Similarly, from $\phi_{\lambda, \mu}^{\prime}(v)=0$ and $\phi_{\lambda, \mu}(v) \leq 0$ one obtains

$$
\left(\frac{1}{2}-\frac{1}{p}\right)\|u\|^{2}+\left(\frac{1}{p}-\frac{1}{q}\right) \lambda\|u\|_{q}^{q} \leq 0 .
$$

This implies that for $\lambda \leq 0$ only $u=0$ is a solution with nonpositive energy. For $\lambda>0$ there are positive constants $c_{3}, c_{4}$ with

$$
c_{3}\|u\|^{2}-\lambda c_{4}\|u\|^{q} \leq 0 \text {, }
$$

hence

$$
\|u\|^{2-q} \leq \lambda c_{4} / c_{3} \rightarrow 0 \quad \text { as } \lambda \rightarrow 0^{+} \text {. }
$$

\section{HAMILTONIAN SYSTEMS}

We consider the Hamiltonian system with periodic conditions

$$
\begin{aligned}
\dot{u}(t) & =J \nabla H_{\lambda, \mu}(u(t)), \\
u(0) & =u(1),
\end{aligned}
$$

where $H_{\lambda, \mu}$ is defined on $\mathbb{R}^{2}$ by

$$
H_{\lambda, \mu}(u):=\frac{\lambda}{q}|u|^{q}+\frac{\mu}{p}|u|^{p}
$$

and $1<q<2<p<\infty$. We denote by $J$ the symplectic matrix $\left(\begin{array}{cc}0 & -1 \\ 1 & 0\end{array}\right)$. Following the Poincare principle, the solutions of (2) are the critical points of the functional

$$
\phi_{\lambda, \mu}(u):=\frac{1}{2}\langle\dot{u}, J u\rangle-\int_{\mathbb{T}} H_{\lambda, \mu}(u(t)) d t
$$

defined on the Sobolev space $X:=H^{1 / 2}\left(\mathbb{T}, \mathbb{R}^{2}\right)$, where $\mathbb{T}:=\mathbb{R} / \mathbb{Z}$. The bracket is the duality bracket between $H^{-1 / 2}\left(\mathbb{T}, \mathbb{R}^{2}\right)$ and $X$.

Theorem 3. Assume that $1<q<2<p<\infty$.

(a) For every $\mu>0, \lambda \in \mathbb{R}$, problem (2) has a sequence of solutions $\left(u_{k}\right)$ such that $\phi_{\lambda, \mu}\left(u_{k}\right) \rightarrow \infty$ as $k \rightarrow \infty$.

(b) For every $\lambda>0, \mu \in \mathbb{R}$, problem (2) has a sequence of solutions $\left(v_{k}\right)$ such that $\phi_{\lambda, \mu}\left(v_{k}\right)<0$ and $\phi_{\lambda, \mu}\left(v_{k}\right) \rightarrow 0$ as $k \rightarrow \infty$.

Proof. Part (a) is a particular case of Theorem 4.1 in [5]. We prove part (b) by using Theorem 2. We assume $\lambda>0$ and set $X(j):=\operatorname{span}\left(e^{2 \pi j t J} \cdot\left(\begin{array}{l}1 \\ 0\end{array}\right)\right)$. Each function $u \in X$ has a Fourier expansion

$$
u \sim \sum_{j \in \mathbb{Z}} e^{2 \pi j t J} \hat{u}(j)
$$


and

$$
\sum_{j \in \mathbb{Z}}|j| \cdot|\hat{u}(j)|^{2}<\infty .
$$

On $X$ we choose the inner product

$$
(u, v):=2 \pi \sum_{j \in \mathbb{Z}}|j| \cdot \hat{u}(j) \overline{\hat{v}(j)}+\hat{u}(0) \overline{\hat{v}(0)}
$$

and the corresponding norm $\|u\|:=\sqrt{(u, u)}$. On each $X(j)$ we consider the antipodal action of $\mathbb{Z} / 2$ so that $\left(A_{1}\right)$ is satisfied.

It is easy to verify $\left(A_{2}\right)$ and $\left(A_{3}\right)$ as in the proof of Theorem 1 . To prove $\left(\mathrm{A}_{4}\right)$, we use the orthogonal decomposition $X=X^{+} \oplus X^{0} \oplus X^{-}$which refers to the subspaces with $j \geq 1, j=0, j \leq-1$ respectively. On $X^{k}$, we have for $\|u\|$ small enough

$$
\begin{aligned}
\phi(u) & \leq \frac{1}{2}\left\|u^{+}\right\|^{2}-\frac{1}{2}\left\|u^{-}\right\|^{2}-\frac{\lambda}{q}\|u\|_{q}^{q}+\frac{|\mu|}{p}\|u\|_{p}^{p} \\
& \leq \frac{1}{2} c\left\|u^{+}\right\|_{q}^{2}-\frac{1}{2}\left\|u^{-}\right\|^{2}-\frac{\lambda}{2 q}\|u\|_{q}^{q} .
\end{aligned}
$$

We have used the fact that $X^{k} \cap X^{+}$is finite dimensional.

Since $\left\|u^{+}\right\|_{q} \leq c_{1}\|u\|_{q}$ on $X^{k} \cap X^{+}$and since $q<2$, we obtain finally for $\|u\|$ small enough,

$$
\begin{aligned}
\phi(u) & \leq c_{2}\|u\|_{q}^{2}-\frac{1}{2}\left\|u^{-}\right\|^{2}-\frac{\lambda}{2 q}\|u\|_{q}^{q} \\
& \leq-\frac{1}{2}\left\|u^{-}\right\|^{2}-c_{3}\|u\|_{q}^{q}
\end{aligned}
$$

where $c_{3}>0$. We choose $r_{k} \in\left(0, R_{k}\right)$ small enough so that the above inequality applies for $\|u\|=r_{k}$. Then, if $\|u\|=r_{k}$ and $\left\|u^{-}\right\| \geq r_{k} / 4$, we have

$$
\phi(u) \leq-r_{k}^{2} / 32 \text {. }
$$

If $\|u\|=r_{k}$ and $\left\|u^{-}\right\| \leq r_{k} / 4$, we have

$$
\phi(u) \leq-c_{3}\left(\left\|u^{+}+u^{0}\right\|_{q}-\left\|u^{-}\right\|_{q}\right)^{q} \leq-c_{3}\left(r_{k} / 2\right)^{q} .
$$

Hence assumption $\left(\mathrm{A}_{4}\right)$ is satisfied.

If $\mu \neq 0$, the proof of the Palais-Smale condition can be found in [10]. If $\mu=0$, the argument is similar.

Remark. Theorem 3 remains true if we consider the Hamiltonian

$$
H_{\lambda, \mu}(u)=a(t)\left(\frac{\lambda}{q} \mu|u|^{q}+\frac{\mu}{p}|u|^{p}\right)
$$

where $a \in \mathscr{C}(\mathbb{T}, \mathbb{R})$ is positive.

\section{ACKNOWLEDGMENT}

The existence of a sequence of positive critical values of $\phi_{\lambda, \mu}$ in Theorem 1 was mentioned to us by Miguel Ramos. 


\section{REFERENCES}

1. A. Ambrosetti, H.Brézis, and G. Cerami, Combined effects of concave and convex nonlinearities in some elliptic problems, J. Funct. Anal. 122 (1994), 519-543.

2. A. Ambrosetti and P. H. Rabinowitz, Dual variational methods in critical point theory and applications, J. Funct. Anal. 14 (1973), 349-381.

3. T. Bartsch, Infinitely many solutions of a symmetric Dirichlet problem, Nonlinear Anal. T.M.A. 20 (1993), 1205-1216.

4. __ Topological methods for variational problems with symmetries, Lecture Notes in Math., vol. 1560, Springer, Berlin and Heidelberg, 1993.

5. T. Bartsch and M. Willem, Periodic solutions of non-autonomous Hamiltonian systems with symmetries, J. Reine Angew. Math. 451 (1994), 149-159.

6. V. Benci, On critical point theory for indefinite functionals in the presence of symmetries, Trans. Amer. Math. Soc. 274 (1982), 533-572.

7. J. Garcia Azorero and I. Peral Alonso, Multiplicity of solutions for elliptic problems with critical exponent or with a nonsymmetric term, Trans. Amer. Math. Soc. 323 (1991), 877-895.

8. P. H. Rabinowitz, Minimax methods in critical point theory with applications to differential equations, CBMS Regional Conf. Ser. in Math., vol. 65, Amer. Math. Soc., Providence, RI, 1986.

9. M. Willem, Lectures in critical point theory, Trabalho de Mat. 199, Fundaçao Univ. Brasilia, Brasilia, 1983.

10. _ Un lemme de déformation quantitatif en calcul des variations, Recherches Mathématiques 19, Université Catholique de Louvain, Louvain-la-Neuve, 1992.

Mathematisches Institut, Universität Heidelberg, Im Neuenheimer Feld 288, 69120 HEIDELBERG, GeRMANY

E-mail address: bartsch@harmless.mathi.uni-heidelberg.de

Institut de Mathématique Pure et Appliquée, Universite Catholique de louvain, Chemin du Cyclotron 2, 1348 Louvain-LA-Neuve, Belgium

E-mail address: willemeamm.ucl.ac.be 